\title{
SERVIÇO SOCIAL E SAÚDE MENTAL: ATUAÇÃO DO ASSISTENTE SOCIAL NA EQUIPE MULTIPROFISSIONAL
}

\section{ARTIGO ORIGINAL}

SILVA, Juliana Cândido da ${ }^{1}$

SANTOS, Henrique Mendes dos ${ }^{2}$

SILVA, Juliana Cândido da. SANTOS, Henrique Mendes dos. Serviço Social e Saúde Mental: Atuação do Assistente Social na equipe multiprofissional. Revista Científica Multidisciplinar Núcleo do Conhecimento. Ano 04, Ed. 10, Vol. 01, pp. 106 120. Outubro de 2019. ISSN: 2448-0959, Link de acesso: https://www.nucleodoconhecimento.com.br/ciencias-sociais/servico-social

\section{RESUMO}

O presente estudo objetiva refletir, teoricamente, sobre a atuação do assistente social inserido em uma equipe multiprofissional dentro da saúde mental, enfatizando a prática interdisciplinar dentro do novo modelo de assistência à saúde mental, o estudo abrange o processo histórico da Reforma Psiquiátrica e a inserção do Serviço Social no campo da saúde mental, explorando também o contexto e a importância da intersetorialidade, integralidade e interdisciplinaridade no referido campo. Utilizou-se, como metodologia, a pesquisa bibliográfica e descritiva baseada na atuação do assistente social dentro de uma unidade psiquiátrica. Destaca, ainda, o papel fundamental da ação profissional no processo de recuperação dos pacientes visando otimizar a reinserção social desses indivíduos.

Palavras-chave: Saúde mental, interdisciplinaridade, serviço social.

\footnotetext{
${ }^{1}$ Pós-graduada em Gestão de Políticas Sociais e Graduada em Serviço Social.

2 Mestrado em Serviço Social; Especialização em Assistência Social e Direitos Humanos; Graduação em Serviço Social.
} 


\section{INTRODUÇÃO}

Segundo a Conferência Regional de Reforma dos Serviços de Saúde Mental (2005), a Reforma Psiquiátrica, no Brasil, evocou mudanças expressivas, sobretudo no modelo de assistência à saúde mental modificou o modelo de assistência à saúde mental trazendo avanços no tratamento à pessoa com transtorno mental, apresentando, portanto, diversas demandas voltadas ao Serviço Social. Todavia, o tema é bastante complexo, visto que carrega marcas do antigo modelo psiquiátrico bem como o preconceito enraizado na sociedade frente às pessoas com transtornos mentais. O Polo de Atenção Integral (PAl) à saúde mental é uma unidade psiquiátrica privada situada no Hospital São Francisco na Providência de Deus, localizado na zona norte da cidade do Rio de Janeiro, tem como função oferecer um atendimento emergencial 24h, com internações de curto prazo para pacientes em crise, contribuindo, assim, com a reinserção do paciente na sociedade a partir de um acompanhamento integral, intersetorial e humanizado.

Durante a internação os pacientes e seus familiares são acompanhados pela equipe multiprofissional do PAI composta por Psiquiatras, Psicólogas, Assistente Social, Terapeutas Ocupacionais, Nutricionista, Educador Físico e Clinico Geral. O movimento de Reforma Psiquiátrica tem como objetivo evidenciar a importância da inserção do assistente social nos serviços de saúde mental, sendo assim é essencial à discussão sobre a atuação da categoria nesse espaço de trabalho, especialmente no âmbito intersetorial e interdisciplinar, a realidade dos pacientes que são atendidos no PAl exige uma intervenção profissional crítica, competente e humanizada, que só poderá se concretizar através de uma análise adequada da realidade, o objetivo do trabalho é apresentar reflexões sobre a intervenção do assistente social no espaço interdisciplinar dentro saúde mental.

A metodologia defendida para a execução do estudo se tratou da pesquisa bibliográfica e descritiva. Nesse contexto, as motivações que levaram a construção deste artigo estão, estreitamente, vinculadas à vivência de atividades desenvolvidas na área em questão. 


\section{DESENVOLVIMENTO}

\section{SAÚDE MENTAL E A REFORMA PSIQUIÁTRICA}

A Organização Mundial de Saúde (OMS) alude que é inexistente a presença de definições advindas de órgãos oficiais frente à saúde mental. Isso ocorre, principalmente, devido às influências culturais e subjetivas. Nesse sentido, Staub e Hoch (2013) entendem, em seu estudo, que a saúde mental deve ser encarada como um estado de bem-estar emocional, e, também, psicológico. Dessa forma, o sujeito deve ser capaz de fazer uso de distintas habilidades emocionais e cognitivas assim como deve desempenhar funções sociais diversas, e, ainda, deve responder às solicitações múltiplas que a rotina cotidiana pede. Para Staub e Hoch (2013) e Lima e Neto (2005), o início do processo da Reforma Psiquiátrica, no país, originou-se a partir do "Movimento Sanitário", nos anos de 1970.

Assim sendo, atuava em favor da mudança dos modelos de atenção e gestão voltados à saúde, à defesa da saúde coletiva e à equidade na oferta de serviços. Pode-se compreender a referida reforma como um processo político e social complexo, visto que tomou forma a partir de atores, instituições e forças de origens múltiplas. Staub e Hoch (2013) e Lima e Neto (2005) enfatizam que devido a este caráter, incidiu em todas as esferas: municipal, estadual e federal bem como nas universidades, no mercado de serviços de saúde, nos conselhos profissionais, nas associações de pessoas com transtornos mentais, nos movimentos sociais diversos e nos domínios marcados pelo imaginário social e pela opinião pública. A Conferência Regional de Reforma dos Serviços de Saúde Mental (2005) foi bastante importante neste contexto.

Ela, por sua vez, compreende um conjunto que tomou forma a partir de transformações de práticas, saberes e valores. Dessa forma, defende que é no cotidiano, ou seja, na vida das instituições, serviços e relações interpessoais que a Reforma Psiquiátrica deve agir. Assim sendo, tratou-se de um processo marcado por impasses, tensões, conflitos e interesses distintos. No contexto brasileiro, a reforma teve o seu ensejo no ano de 1978. Tratou-se de um movimento social em busca pelos direitos dos pacientes psiquiátricos. A luta ficou conhecida como "Movimento dos 
Trabalhadores em Saúde Mental" (STAUB; HOCH, 2013; LIMA; NETO, 2003; AMARANTE; NUNES, 2018). Foi formado por trabalhadores integrantes do Movimento Sanitário, por familiares dos pacientes, por sindicalistas e por pessoas com histórico de internações psiquiátricas.

A literatura aponta que a partir desse movimento bem como por meio de lutas diversas, foram denunciados diversos casos de violência nos manicômios, e, também, criticou-se, com afinco, o modelo hospitalocêntrico voltado à assistência de pessoas com transtornos mentais (STAUB; HOCH, 2013; LIMA; NETO, 2003; AMARANTE; NUNES, 2018). Foi nesse contexto que surgiram as primeiras propostas e sugestões de ações a serem implementadas para reorientar a assistência a esses indivíduos portadores de transtornos mentais. Pautado nesses ideais, no ano de 1987, a $2^{\mathrm{a}}$ edição do Congresso Nacional do MTSM, realizado em Bauru, no estado de São Paulo, adotou o lema "por uma sociedade sem manicômios".

Os estudos de Carvalho (2010) e Souza (2015) apontam que, ainda em 1987, foi realizada a $1^{\underline{a}}$ edição da Conferência Nacional de Saúde Mental, no Rio de Janeiro. Surgiu, ainda, neste período, o primeiro CAPS no país. A sua sede foi construída em São Paulo, no ano de 1987. No ano de 1989 teve ensejo um processo de intervenção da Secretaria Municipal de Santos (São Paulo) na Casa de Saúde Anchieta. A principal motivação para a intervenção foi que este hospital era um local de maus tratos de pacientes e havia, ainda, mortes. Dessa forma, em Santos, foram implementados os Núcleos de Atenção Psicossocial (NAPS). Esses oferecem um atendimento de 24 horas aos pacientes. A experiência de Santos passou, então, a ser um marco para o processo de reforma na psiquiatria brasileira.

Assim, no ano de 1989, foi instituído um projeto de lei proposto pelo Deputado Paulo Delgado, do PT/MG. Deu-se entrada deste no Congresso Nacional. A Lei propunha a regulamentação dos direitos da pessoa portadora de transtornos mentais bem como defende a extinção progressiva dos manicômios no Brasil. Os estudos de Carvalho (2010) e Souza (2015) enfatizam que este foi o início das lutas do Movimento da Reforma Psiquiátrica nos âmbitos legislativo e normativo. No ano de 1992, movimentos sociais inspirados pelo referido projeto de lei, conseguiram aprovar, em 
vários estados brasileiros, as primeiras leis que defendiam a substituição progressiva dos leitos psiquiátricos por uma rede integrada de atenção à saúde mental (MELGAÇO, 2013; CARVALHO, 2010; FILHO et al, 2015; ALVAREZ; MARTINS, 2012).

Foi neste período, também, que a política do Ministério da Saúde voltada à saúde mental passou a acompanhar as diretrizes da Reforma Psiquiátrica (MELGAÇO, 2013; CARVALHO, 2010; FILHO et al, 2015; ALVAREZ; MARTINS, 2012). Dessa forma, ela passou a ganhar diretrizes melhores definidas. A década de 1990 é marcada pelo compromisso firmado pelo país com a Declaração de Caracas, promovida na $2^{\mathrm{a}}$ edição da Conferência Nacional de Saúde Mental. Passam, então, a vigorar, no país, as primeiras normas federais que regulamentavam a implementação de serviços voltados à atenção diária com base na experiência dos primeiros CAPS, NAPS e Hospitais-Dia. Surgiram, também, as primeiras normas de fiscalização e classificação dos hospitais psiquiátricos.

No ano de 2001, após 12 anos de trâmite no Congresso Nacional, a Lei proposta por Paulo Delgada foi sancionada. Contudo, a aprovação se tratou de um substitutivo para o projeto de lei original, visto que trouxe modificações importantes em seu texto normativo (FONTE, 2012; DIMENSTEIN et al, 2009; SOUZA, 2015; ALVAREZ; MARTINS, 2012; GONZALEZ, 2015; OLIVEIRA, 2013). Destarte, a Lei Federal de 2016 redirecionou a assistência em saúde mental. Houve, assim, certo privilégio no oferecimento de tratamento em serviços de base comunitária. Assim, dispõe sobre a proteção aos direitos das pessoas portadoras de transtornos mentais, contudo não institui mecanismos claros frente à progressiva extinção dos manicômios. Houve, também, a desinstitucionalização de pessoas com internação de longa duração.

Este processo foi impulsionado pela criação do "Programa De Volta para Casa". Foi estabelecida, também, uma política de recursos humanos para que a Reforma Psiquiátrica fosse construída (FONTE, 2012; DIMENSTEIN et al, 2009; SOUZA, 2015; ALVAREZ; MARTINS, 2012; GONZALEZ, 2015; OLIVEIRA, 2013). Traçou-se, então, políticas voltadas ao álcool e outras drogas. Para tanto, incorporou-se a estratégia para a redução de danos. No ano de 2004 foi realizado o primeiro Congresso Brasileiro 
de Centros de Atenção Psicossocial, em São Paulo. Reuniu-se dois mil trabalhadores e usuários do CAPS. Assim, com a implementação bem como com o financiamento dos Serviços Residenciais Terapêuticos (SRT) surgiram em detrimento do processo de desinstitucionalização e como componentes importantes para políticas de saúde mental.

Surgiram, sobretudo, para superar o modelo de atenção centrado no hospital psiquiátrico. Nesse sentido, os SRT's, as residências terapêuticas ou, simplesmente, as moradias, são casas localizadas no espaço urbano que têm como objetivo prestar assistência àqueles portadores de transtornos mentais e que, dessa forma, precisam de ajuda frequentemente, sejam eles oriundos de hospitais psiquiátricos ou não. Em síntese, com a aprovação da Lei de $\mathrm{N}^{\circ} 10.216 / 01$, deu-se origem à Portaria de $\mathrm{N}^{\circ}$ 336/MG, em 19 de fevereiro de 2002. Esta, por sua vez, regulamentou os serviços para a substituição do modelo hospitalocêntrico. Com essas mudanças na forma de tratamento, o "doente" mental/paciente, como era até então chamado, passa a ser considerado como um usuário sujeito de direitos, e, assim, digno de tratamento.

É possível concluir este capítulo afirmando que buscou-se por novas alternativas voltadas à assistência à saúde mental (FONTE, 2012; DIMENSTEIN et al, 2009; SOUZA, 2015; ALVAREZ; MARTINS, 2012; GONZALEZ, 2015; OLIVEIRA, 2013) desde a criação dos CAPS, NAPS, dos Hospitais-Dia e das Residências Terapêuticas. Dessa forma, o novo conceito de saúde mental passou a priorizar o sujeito e não mais a doença em si. Assim, a esse sujeito, passou a ser oferecido um tratamento mais humano e de qualidade. Criou-se, para tanto, aparatos, dispositivos e espaços apropriados direcionados ao seu tratamento. Precisa-se, então, aderir a ações intersetoriais bem como às políticas sociais diversas de forma a possibilitar uma intervenção interdisciplinar que visa a integralidade ao atender o sujeito portador de transtornos mentais. 


\section{INTERSETORIALIDADE, INTERDISCIPLINARIDADE} INTEGRALIDADE

Com a promulgação das Leis 8.080 e 8.142, ambas do ano de 1997, foi implementado, no Brasil, o Sistema Único de Saúde (SUS). Este, por sua vez, tem como base o Art. 198 da Constituição Federal de 1988 (BREDOW; DRAVANZ, 2010; SCHÜTZ, 2009; APPEL, 2017). As diretrizes principais do SUS tratam-se da universalidade e integralidade deste no atendimento aos usuários. Defende, também, a descentralização dos recursos bem como a regionalização. Visa, ainda, atender, de forma singular, cada região. Nesse sentido, a concepção de saúde ultrapassa a ideia simplório de "ausência da doença". Dessa forma, a Política de Saúde, no Brasil, organiza-se de forma intersetorial para que seja possível enfrentar os fatores diversos que se manifestam no processo de adoecimento por meio, sobretudo, de ações integradas.

A saúde tem como fatores determinantes e condicionantes, entre outros, a alimentação, a moradia, o saneamento básico, o meio ambiente, o trabalho, a renda, a educação, o transporte, o lazer e o acesso aos bens e serviços essenciais; os níveis de saúde da população expressam a organização social e econômica do País (BRASIL, 1990).

De acordo com a literatura, os aspectos sociais, econômicos e culturais de uma nação repercutem, diretamente, nos níveis de saúde de uma dada população (CAMPOS; CASTRO, 2004; BREDOW; DRAVANZ, 2010; SCHÜTZ, 2009; APPEL, 2017). Nesse contexto, a saúde pode ser definida como o resultado dos modos de organização social da produção. Tem-se, então, como efeito, os mais diversos fatores. Esses, por sua vez, exigem, do Estado, que este assuma a responsabilidade pela execução de políticas de saúde integradas às demais políticas sociais e econômicas exigidas pela população. Dessa forma, o Estado tem o dever de garantir a sua efetivação bem como cabe a ele, também, fazer com que o setor da saúde promova condições de vida mais dignas por meio, principalmente, do exercício pleno da cidadania.

Todavia, é preciso enfatizar, com este estudo e de acordo com a literatura, que é de extrema importância, a criação, por parte do Estado, de dispositivos, aparatos, 
ferramentas, metodologias e semelhantes para o combate efetivo bem como para a erradicação dos fatores que fazem com que uma ou mais pessoas adoeçam (CAMPOS; CASTRO, 2004; BREDOW; DRAVANZ, 2010; SCHÜTZ , 2009; APPEL, 2017). Deve-se, ainda, além da efetivação das políticas de saúde integradas às demais, é preciso caminhar em busca da intersetorialidade das políticas de saúde com as demais demandas sociais. Assim, diferentes mecanismos devem ser acionados para que o trabalho compartilhado seja enfatizado no processo de enfrentamento dos problemas sociais. A intersetorialidade, para Campos e Castro (2004, p. 74) é:

Nada mais do que um processo de construção compartilhada, em que os diversos setores envolvidos são tocados por saberes, linguagens e modos de fazer que não lhes são usuais, pois pertencem ou se localizam no núcleo da atividade de seus parceiros. A intersetorialidade implica a existência de algum grau de abertura em cada setor envolvido para dialogar, estabelecendo vínculos de corresponsabilidade e cogestão pela melhoria da qualidade de vida da população.

Nessa perspectiva, considerar o cidadão de forma íntegra, ou seja, ser sensível às suas necessidades individuais e coletivas, colabora para com a execução de ações efetivas voltadas à promoção da saúde (CEZAR; MIOTO; SCHUTZ, 2008; DIAS, 2010; CAMPOS; CASTRO, 2004; BREDOW; DRAVANZ, 2010; SCHÜTZ, 2009; APPEL, 2017). Deve-se enfatizar, também, que a construção de parcerias com outros setores, ou seja, com as esferas da educação, do trabalho, do emprego, da habitação, da cultura, da segurança, do alimento é essencial. Assim, a promoção da saúde depende, sobretudo, da satisfação de diversas necessidades sociais que se manifestam por meio de políticas e demandas. Essas não são diferentes no setor da saúde. É necessário, portanto, o envolvimento com outras categorias para que fatores negativos no processo de saúde/doença sejam solucionados.

No domínio da saúde mental, a integralidade do cuidado é um princípio ético e político que implica na organização e efetivação do atendimento e prestação de serviços de forma que o usuário seja atendido de maneira integral (CEZAR; MIOTO; SCHUTZ, 2008; DIAS, 2010; CAMPOS; CASTRO, 2004; BREDOW; DRAVANZ, 2010; SCHÜTZ, 2009; APPEL, 2017). Busca-se, então, superar a fragmentação na prestação do atendimento. Para isso é necessária uma compreensão ampla sofre os fatores que 
fazem com que o sujeito adoeça. Nesse sentido, a Política Nacional de Promoção à Saúde defende que a integralidade deve caminhar para além da articulação de estratégias direcionadas à produção da saúde. Deve haver, portanto, uma comunicação mais ampla para que as necessidades individuais e coletivas dos usuários sejam melhores compreendidas.

É preciso, contudo, desvincular-se uma perspectiva estrita que enfoca, apenas, no adoecimento bem como nos sintomas e aderir a uma vertente que se preocupe com o estudo da história do paciente, de suas condições de vida e de suas necessidades em termos de saúde (CEZAR; MIOTO; SCHUTZ, 2008; DIAS, 2010; CAMPOS; CASTRO, 2004; BREDOW; DRAVANZ, 2010; SCHÜTZ, 2009; APPEL, 2017). Devese, então, compreender que a integralidade não se trata, apenas, de um conceito. É uma diretriz que se ser adotada diariamente. Esse processo pode ser alcançado por meio da interdisciplinaridade. Esta, por sua vez, precisa enfocar em métodos que enfatizam a reconstrução da atuação do assistente social. Apresentar objetivos e estratégias para tornar esse atendimento mais eficiente é essencial. $O$ trabalho em equipe é um poderoso aliado para se chegar à interdisciplinaridade.

Para o alcance de bons resultados, as equipes precisam ser compostas a partir de profissionais de áreas distintas, e, assim, deve-se respeitar as suas especificidade bem como é preciso garantir a multidisciplinaridade de suas tendências para que haja a troca de experiências (CEZAR; MIOTO; SCHUTZ, 2008; DIAS, 2010; CAMPOS; CASTRO, 2004; BREDOW; DRAVANZ, 2010; SCHÜTZ, 2009; APPEL, 2017). Nessa perspectiva, os desafios mais recorrentes na prática do assistente social se voltam, principalmente, à concretização das políticas sociais. Esses devem, então, proporcionar um melhor acesso às redes institucionais para que os usuários se sintam amparados nas mais diversas esferas. No domínio da Saúde Mental, deve-se garantir a efetivação dos direitos a partir de redes de apoio que envolvem equipes multidisciplinares.

A partir dos estudos de Vasconcelos (2008) e de Machado (2007) é possível concluir este capítulo afirmando que a multidisciplinaridade deve ser concebida a partir de práticas de desinstitucionalização. Assim, as instituições devem aderir a ações mais 
humanizadas no processo de tratamento terapêutico. Nesse sentido, o trabalho do assistente social que presta assistência à Saúde Mental, deve compreender atitudes interdisciplinares. Esta, por sua vez, implica no desenvolvimento de diversas competências, habilidades, saberes e práticas. Deve-se, portanto, priorizar os princípios da integralidade, da intersetorialidade e da contabilidade para que seja possível contemplar ou aproximar, ao máximo, de indivíduos e/ou grupos de forma íntegra e ampla.

\section{ATUAÇÃO DO ASSISTENTE SOCIAL EM EQUIPE MULTIDISCIPLINAR NA SAÚDE MENTAL}

A esfera da Saúde Mental é um espaço bastante rico para atuação profissional, em que 0 assistente social trabalha com outros profissionais a partir de uma perspectiva interdisciplinar. Conforme discutido anteriormente, a interdisciplinaridade implica uma atuação conjunta das diferentes áreas do saber, a mistura desses saberes e o desafio de preservar a particularidade de cada profissão. O Código de Ética do Assistente Social, de forma conjunta com a Lei da Reforma Psiquiátrica, destaca a necessidade de se enfatizar a interdisciplinaridade tanto na atuação profissional quando na prestação integral da saúde mental I ao usuário, a qual rompe com a fragmentação do atendimento. Assim, enfatiza-se que a definição de uma saúde ampla compreende na busca por um diálogo entre as diferentes categorias profissionais, desde o ensino até a materialização das ações.

Conforme Vasconcelos (2008), a Reforma Psiquiátrica rompeu, expressivamente, com os saberes tradicionais, e, assim, abriu espaço para a prática de ações de caráter interdisciplinar. $O$ estudo em questão alude, também, que embora cada profissão possua uma caracterização própria, em termos de cultura, prática, estratégias e saberes diferenciados, é preciso que as equipes busquem, continuamente, pela reelaboração de uma cultura profissional mais flexível e aberta a prática do trabalho de forma interdisciplinar. As reflexões deste artigo são baseadas na atuação do Serviço Social na equipe multidisciplinar do Polo de Atenção Integral (PAI) à Saúde Mental, setor do Hospital São Francisco na Providência de Deus, o público alvo deste 
setor são pessoas com transtornos mentais e dependentes químicos em momentos de crise.

Atualmente o hospital é administrado pelos frades da Associação e Fraternidade São Francisco de Assis na Providência de Deus. É um hospital bastante importante de ser mencionado, visto que fornece, aos pacientes, um tratamento intersetorial e integral. Assim sendo, defende-se que o espaço sócio ocupacional da saúde mental, representado pelo Serviço Social, deve se ater a todas as expressões sociais, sobretudo daquelas que surgem a partir da exclusão da pessoa portadora de transtornos mentais. Deve-se enfatizar que, durantes muitos anos, esses sujeitos, eram rotulados como pessoas perigosas e incapazes, e, portanto, deveriam ser excluídas da sociedade e mantidas, definitivamente, em manicômios. Infelizmente, mesmo nos dias atuais, o estigma social e o preconceito rondam a vida desses indivíduos, bem como, de seus familiares.

A reforma psiquiátrica abre um campo oportuno para o Serviço Social devido à sua organização social política, apresentando a necessidade de os profissionais estarem atuando nessa área. Bisneto (2009) observa os desafios atuais presentes na prática do Serviço Social na área de saúde mental. Destacando avanços significativos, relacionados à viabilização de saberes e metodologias que incentivam o fazer profissional. Para Bisneto (2009), o profissional precisa articular, não somente com a categoria profissional, mas também com a equipe multidisciplinar, ampliando uma visão crítica acerca do processo de trabalho em saúde mental, da realidade social, da relação entre o desenvolvimento do capitalismo e a existência crescente de transtornos mentais e uso de substâncias psicoativas.

Exige-se, pois, a apreensão do sujeito em sua integralidade, considerando sua relação com o meio em que está inserido. Alguns dos objetivos do Serviço Social na saúde mental, especialmente, durante um período de internação do paciente, trabalhando em conjunto com a equipe multiprofissional, são: conhecer e analisar a realidade social do paciente, com o objetivo de identificar, de maneira crítica, as manifestações da questão social presente na vida desse indivíduo; desenvolver estratégias de intervenção juntamente com os familiares de pacientes, a fim de fortalecer os vínculos 
familiares; identificar e consolidar mecanismos de suporte e proteção, buscando a reinserção social dos usuários, resgate da cidadania e a vivência de hábitos saudáveis; buscar métodos que permitam identificar os direitos dos pacientes e que possibilitem a defesa e a universalização deles.

$\mathrm{Na}$ prática profissional o assistente social realiza sua intervenção no âmbito das relações sociais, assim, para um desempenho efetivo, deve buscar uma visão ampla, dada por meio de uma leitura da realidade. Vasconcelos (2012) destaca que o assistente social sinaliza os determinantes sociais e estabelece relações e conexões necessárias entre as diferentes especialidades profissionais e a questão social do indivíduo que está sendo atendido, no entanto, apesar de as expressões da questão social serem objeto de intervenção do assistente social, não devem ser objeto de atenção apenas desta categoria, pois isso limita e prejudica a viabilização de políticas sociais na direção dos interesses e necessidades dos usuários. Assim, "a questão social, se é objeto de preocupação e de ação dos assistentes sociais, não é objeto exclusivo de sua responsabilidade".

Segundo Bisneto (2007) as implicações existentes entre a questão social e a saúde mental se dá em duas direções: os problemas sociais podem desencadear os problemas mentais e o indivíduo que sofre com problemas mentais tem agravada a sua problemática social. $\mathrm{O}$ atendimento do assistente social deve priorizar realização de uma escuta qualificada, ampla, que busque compreender o contexto em que está inserido o sujeito e os conflitos, desafios, questionamentos, medos e dúvidas que $o$ envolvem, não é "psicologizar" o atendimento, mas ir ao encontro com o que propõe o Projeto Ético Político do serviço social. E que atribui novas competências e conhecimento ao agir profissional, viabiliza a captação de informações a fim de compreender o sujeito em sua totalidade.

Para concluir este estudo vale elucidar que o assistente social não deve considerar, apenas, o sujeito ou as suas necessidades aparentes. É preciso que essas pessoas sejam enxergadas para além da doença mental que possuem. Assim, deve-se superar estimas e tornar os serviços da rede de saúde mais articulados com a educação e com a assistência para que a integralidade aqui defendida seja alcançada. A partir do 
defendido com este estudo, entende-se que a relevância do Serviço Social dentro de equipes que atuam de forma multidisciplinar na área da Saúde Mental se expandiu, expressivamente, nos últimos anos, visto que se aumentou a inclusão de usuários a familiares nas políticas sociais. Esses passaram a ser inseridos nas ações cidadãs, e, dessa forma, passaram a possuir um melhor acesso aos direitos. Essas demandas, contudo, precisam ser motivadas pelo assistente social.

\section{CONCLUSÃO}

As demandas das pessoas com transtornos mentais e seus familiares são variadas e divergem em diferentes etapas da doença, o que apresenta a necessidade de um atendimento integral, especialmente, nos casos de internação, onde o suporte da equipe multidisciplinar é indispensável. Nesse sentindo a atuação do Serviço Social visa à promoção e a garantia dos direitos sociais das pessoas com transtornos mentais, bem como, de seus familiares. O atual debate do Serviço Social na saúde mental foi estimulado, principalmente pelo processo de Reforma Psiquiátrica brasileira, visto que a partir deste movimento surge uma nova pratica profissional a fim de atender os indivíduos em sofrimento mental em sua totalidade, com objetivo de resgatar os direitos dessas pessoas.

Cabe aos profissionais da saúde mental, sobretudo, ao assistente social considerar o contexto de vida do sujeito e assim aumentar a qualidade do atendimento, para isso a equipe multiprofissional deve estar comprometida ética e politicamente com a Reforma Psiquiátrica, garantido tratamento humanizado e a reinserção social do paciente. A partir dos dispositivos teórico-metodológicos, ético-políticos e técnicooperativos, o assistente social tem as ferramentas necessárias para a construção e articulação de diversos espaços de atenção ao usuário, bem como, o desenvolvimento de ações que visem à melhoria dos serviços, o aumento da participação e a autonomia dos pacientes.

Portanto, contribuir com reflexões acerca do trabalho do assistente social em equipe multiprofissional na saúde mental, possibilita novos debates sobre este tema, que ainda é tão complexo de ser discutido, porém, essa discursão é extremamente 
importante para promover e fortalecer a integralidade no atendimento, o desenvolvimento da autonomia das pessoas com transtornos mentais, a participação popular e, consequentemente, a melhoria da qualidade de vida do paciente e a superação de expressões da questão social.

\section{REFERÊNCIAS}

ALVAREZ, C. R. S; MARTINS, M. B. A terapia ocupacional e suas possíveis contribuições na saúde mental coletiva. VITTALLE-Revista de Ciências da Saúde, v. 24, n. 2, p. 63-68, 2012.

AMARANTE, P; NUNES, M. de. O. A reforma psiquiátrica no SUS e a luta por uma sociedade sem manicômios. Ciência \& Saúde Coletiva, v. 23, n. 6, p. 2067-2074, 2018.

APPEL, N. M. O assistente social inserido na saúde mental e suas estratégias de intervenção. In: VIII Jornada Internacional de Políticas Públicas, 2017. Disponível em:http://www.joinpp.ufma.br/jornadas/joinpp2017/pdfs/eixo8/oassistentesocialinseri donasaudementalesuasestrategiasdeintervencao.pdf. Acesso em: 18 de set 2019.

BISNETO, J. A. Serviço Social e Saúde Mental: uma análise institucional da prática. 2. ed. São Paulo: Cortez, 2009.

BREDOW, S. G; DRAVANZ, G. M. Atuação do Serviço Social na Saúde Mental: entre os desafios e perspectivas para efetivação de uma política intersetorial, integral e resolutiva. Textos \& Contextos (Porto Alegre), v. 9, n. 2, p. 229-243, 2010.

CAMPOS, R. B. de; CASTRO, A. M. de. Avaliação de política nacional de promoção da saúde. Ciências e Saúde Coletiva, v. 9, n. 3, p. 745-749, 2004.

CARVALHO, A. Z. T. de. Produção do cuidado em saúde mental: uma cartografia da rede de atenção em Fortaleza - CE. Dissertação de Mestrado em Enfermagem Universidade Estadual de Ceará. Fortaleza, 2010. Disponível em: 
http://www.uece.br/cmacclis/index.php?option=com_content\&view=article\&id=69\&lte mid=144. Acesso: 17 set 2019.

CEZAR, C. A.; MIOTO, R. C. T.; SCHUTZ, F. A construção da intersetorialidade em saúde como estratégia na garantia de direitos. In: CONFERÊNCIA MUNDIAL DE SERVIÇO SOCIAL, 19, Salvador, 2008. Disponível em: https://doi.org/10.20396/sss.v10i2.8634832. Acesso em: 19 set 2019.

CONFERÊNCIA REGIONAL DE REFORMA DOS SERVIÇOS DE SAÚDE MENTAL. Reforma Psiquiátrica e política de Saúde Mental no Brasil. Ministério da Saúde: Brasília, 2005.

Disponível

em: http://bvsms.saude.gov.br/bvs/publicacoes/Relatorio15_anos_Caracas.pdf. Acesso em: 18 set 2019.

DIAS, M. Saúde mental e políticas de estado: pactuar caminhos intersetoriais. Apresentação proferida na IV Conferência Municipal de Saúde Mental de Pelotas. $\quad 2010.2$ Delotas, Disponível http://bvsms.saude.gov.br/bvs/publicacoes/relatorio_final_4_conferencia_saude_men tal.pdf. Acesso em: 19 set 2019.

FILHO, A. J. de. A. F. et al. Trajetória histórica da reforma psiquiátrica em Portugal e no Brasil. Revista de Enfermagem Referência, n. 4, p. 117-125, 2015.

GONZALEZ, A. M. Plano de ações na promoção e prevenção de transtornos em saúde mental na atenção primária à saúde. Trabalho de Conclusão de Curso em Atenção Básica em Saúde da Família - Universidade Federal de Minas Gerais. Contagem, 2015.

Disponível

em: https://www.nescon.medicina.ufmg.br/biblioteca/imagem/4979.pdf. Acesso em: 17 set 2019.

LIMA, V. B Oliveira; NETO, J. do R. C. B. Reforma psiquiátrica e políticas públicas de saúde mental no Brasil: resgate da cidadania das pessoas portadoras de transtornos mentais. Direito \&Política, v. 1, n. 1, p. 121-31, 2011. 
MACHADO, S. G. A singularidade e a pluralidade do serviço social no processo de trabalho em saúde mental. Dissertação de Mestrado em Serviço Social Universidade Federal do Rio de Janeiro. Rio de Janeiro, 2007.

MELGAÇO, L. de. M. Inserção das pessoas portadoras de transtornos mentais na atenção da equipe de saúde da família. Trabalho de Conclusão de Curso Universidade Federal de Minas Gerais. Lagoa Santa, 2013. Disponível em: https://www.nescon.medicina.ufmg.br/biblioteca/imagem/4380.pdf. Acesso em: 19 set 2019.

OLIVEIRA, D. S. de. Apoio matriarcal e equipe de referência: modos de reinventar as práticas no cotidiano da saúde mental. Trabalho de Conclusão de Curso em Terapia Ocupacional - Universidade de Brasília. Brasília, 2013. Disponível em: http://bdm.unb.br/handle/10489/6930. Acesso em: 16 set 2019.

SOUZA, P. R. P. de. A estratégia de saúde da família e os cuidados de enfermagem em saúde mental. Monografia para o II Curso de Especialização em Saúde Mental - Universidade de Brasília. Brasília, 2015.

SCHÜTZ, F. A intersetorialidade no campo da política pública: indagações para o serviço social. Trabalho de Conclusão de Curso em Serviços Sociais Universidade Federal de Santa Catarina. Florianópolis, 2009. Disponível em: https://repositorio.ufsc.br/handle/123456789/120179. Acesso em: 18 set 2019.

STAUB, M. L; HOCH, V. A. A utilização de psicofármacos no tratamento de saúde mental. Disponível em: http://www.uniedu.sed.sc.gov.br/wpcontent/uploads/2013/10/Maria-Lucia-Staub.pdf. Acesso em: 18 set 2019.

VASCONCELOS, A. M. A prática do serviço social: cotidiano, formação e alternativas na área da saúde. 8. ed. São Paulo: Cortez, 2012.

Enviado: Julho, 2019.

Aprovado: Outubro, 2019. 